\title{
Patient derived Fab structures suggest mechanism by which affinity maturation promotes autoantibody recognition of MuSK in the autoimmune disease myasthenia gravis
}

\author{
C Vieni ${ }^{1,3}$, M Fichtner ${ }^{2}$, R Redler ${ }^{3}$, L Kolich ${ }^{3}$, S Burden ${ }^{3}$, K O'Connor $^{2}$, D Ekiert ${ }^{1}$ \\ ${ }^{1}$ New York University School of Medicine, New York, NY, ${ }^{2}$ Department of Immunobiology, Yale \\ University School of Medicine, New Haven, CT, ${ }^{3}$ New York University Grossman School of \\ Medicine, New York, NY \\ casey.vieni@nyumc.org
}

Myasthenia gravis (MG) is a chronic autoimmune disorder affecting neuromuscular transmission caused by pathogenic autoantibodies targeting components of the neuromuscular junction. Pathogenic autoantibodies against the muscle-specific tyrosine kinase (MuSK) are predominantly of the IgG4 subclass, which have the unique ability to participate in Fab-arm exchange making them functionally monovalent. Recent isolation of human MuSK monoclonal autoantibodies (mAbs) from patients with MG has allowed for the characterization of MuSK-specific autoantibodies. Using X-ray crystallography we have structurally characterized two patient derived Fabs from MG. Our data suggests that affinity maturation increases the negative charge of the CDR loops which increases Fab affinity to the positively charged second Ig-like domain in MuSK. Affinity maturation leads to sub-nanomolar binding to the autoantigen, while unmutated common ancestors (UCA) Fabs have an approximately 100 fold lower affinity than their respective mature fabs. In acetylcholine receptor clustering assays mature mAbs, UCA mAbs, and mature Fabs bound the autoantigen with pathogenic capacity, while monovalent UCA Fabs bound the autoantigen without measurable pathogenic capacity. These findings suggest that high affinity, functionally monovalent, Fab-arm exchanged IgG4 antibodies tightly bind to the autoantigen MuSK in MuSK MG, thereby preventing MuSK dimerization and downstream signaling, rather than receptor cross linking and activation.

Acta Cryst. (2020). A76, a106 